\title{
On the Decidability of the Evolution of the Fuzzy Cellular Automaton 184
}

\author{
Angelo B. Mingarelli ${ }^{1}$ and Samira El Yacoubi ${ }^{2}$ \\ ${ }^{1}$ Mathematics and Statistics, Carleton University, Ottawa, Canada, K1S 5B6 \\ ${ }^{2}$ Laboratory of Mathematics and Physics for Systems (MEPS), University of \\ Perpignan, 52, Paul Alduy Avenue 66860 - Perpignan Cedex. France
}

\begin{abstract}
In the previous paper [1] we presented general methods for detecting the evolution and dynamics of any one of the 255 fuzzy cellular automata (FCA) and showed that the method was applicable to all but nine of the $255 \mathrm{FCA}$. The main result there was that the limiting behavior of these FCA is decidable, except possibly for these nine, for finite initial configurations in a homogeneous background of zeros. Only six of these nine so called exceptional CA namely, FCA 172, 184, 202, 216, 226, and 228 , appear to be interesting enough to warrant separate study, the other three, namely FCA 204, 228, and 240 being trivial. In this paper we study the exceptional FCA 184, a cellular automaton that admits a continuum of fixed points, namely the interval $[0,1]$. This FCA is of interest because the general technique developed in [1] fails for the determination of its asymptotics. We show, in particular, that the asymptotic evolution of FCA 184 from any finite initial including random configuration of nonzero cells is decidable.
\end{abstract}

\section{Introduction}

The elementary cellular automata (ECA), considered by Wolfram and others, are wonderful examples of systems with simple rules that may produce unusually complex behavior; some of them, which are hardly predictable, are even capable of universal computation, 2]. On the other hand, one may get a better sense of their behavior by approximating their dynamics by continuous state CA's (or coupled map lattices), to wit, by "fuzzifying" the disjunctive normal form (the ECA is then called an FCA) and then studying the dynamics of the resulting global function as was done in 3. Recent work in this new area of continuous CA's includes some variations on the game of Life in [4] and applications to pattern recognition [5]. In addition, such CA have been used to investigate the result of perturbations, for example, noisy sources, computation errors, mutations, etc. on the evolution of boolean cellular automata (cf., [6], [3, 7], etc.).

We recall some terminology for ease of exposition: A one-dimensional (boolean or binary-state) cellular automaton is a collection of ones and zeros (called an initial string) arranged on an infinite one-dimensional strip (where the space variable goes in the horizontal direction). Each cell is preceded by a cell behind it and another in front of it; these are called the neighbors of the cell. In the notation 
of [8] these are known as " $k=2, r=1$ CA's". We choose an arbitrary (but fixed) special element of this initial strip (at time $t=0$ ) by labeling it as $x_{0}$ (or by $x_{0}^{0}$ ). Denoting the two-point set (or boolean space) consisting of the numbers 0 and 1 by $\{0,1\}$ we define a local rule (sometimes also called a transition rule) by a function $g:\{0,1\}^{3} \rightarrow\{0,1\}$, on consecutive triples $x_{i-1}, x_{i}, x_{i+1}$ of this strip with the effect that $f(x)_{i}=g\left(x_{i-1}, x_{i}, x_{i+1}\right)$ where the value $f(x)_{i}$ is placed directly below $x_{i}$, where time goes vertically down the diagram (on another such parallel strip which determines the states of the automaton at $t=1$ ) and this update of the original cell values is performed for all the quantities in the initial string resulting in two parallel strips. The procedure is then repeated again and again to generate an infinite sequence of strips whose evolution at infinity we wish to study. The resulting diagram is called a light-cone or space-time diagram, see 8 . It follows that for a given general $\mathrm{CA}$ with transition rule $g$ its states are related by

$$
x_{i}^{t+1}=g\left(x_{i-1}^{t}, x_{i}^{t}, x_{i+1}^{t}\right), \quad t \geq 0 .
$$

Now, in the normal boolean case, each of three consecutive cells can have two values, namely, 0 and 1 . Thus, the automaton is uniquely determined once its values on the eight basic elements 000, 001, 010, 100, 110, 101, 011, 111 are known. So there can be at most $2^{8}=256$ such CA's and these are commonly known as the elementary cellular automata (ECA for short). To distinguish each automaton from every other in this list of several hundred, Wolfram [9] used a numbering scheme based on the binary expansion of each number $n$ in the form $n=\sum_{i=0}^{\infty} r_{i} 2^{i}$, where $0 \leq n \leq 255$ and combined this with the disjunctive normal form. Specifically, every transition rule of such elementary CA's is expressible as a disjunctive normal form (abbr. DNF), e.g.,

$$
g\left(x_{1}, x_{2}, x_{3}\right)=\vee_{i \mid r_{i}=1} \wedge_{j=1}^{3} x_{j}^{d_{i j}}
$$

where $d_{i j}$ is the $j$-th digit, from left to right, of the binary representation of $i$, and $x^{0}$ (resp. $x^{1}$ ) stands for $\neg x$ (resp. $x$ ). This DNF is simply a comprehensive device for the writing of any one of the 256 ECA's. We are particularly interested in ECA 184 and its "fuzzification" defined below. Indeed, since $184=2^{3}+2^{4}+$ $2^{5}+2^{7}$ we see that the so called rule number $184=\sum_{i=0}^{7} r_{i} 2^{i}$ forces $r_{i}=1$ only for $i=3,4,5,7$. Use of the disjunctive normal form expression above gives us

$$
\begin{aligned}
g_{184}\left(x_{1}, x_{2}, x_{3}\right)= & \vee_{i \mid r_{i}=1} \wedge x_{j=1}^{3} x_{j}^{d_{i j}}, \\
= & \left(x_{1}^{d_{31}} \wedge x_{2}^{d_{32}} \wedge x_{3}^{d_{33}}\right) \vee\left(x_{1}^{d_{41}} \wedge x_{2}^{d_{42}} \wedge x_{3}^{d_{43}}\right) \vee\left(x_{1}^{d_{51}} \wedge x_{2}^{d_{52}} \wedge x_{3}^{d_{53}}\right) \\
& \vee\left(x_{1}^{d_{71}} \wedge x_{2}^{d_{72}} \wedge x_{j}^{d_{73}}\right), \\
= & \left(x_{1}^{0} \wedge x_{2}^{1} \wedge x_{3}^{1}\right) \vee\left(x_{1}^{1} \wedge x_{2}^{0} \wedge x_{3}^{0}\right) \vee\left(x_{1}^{1} \wedge x_{2}^{0} \wedge x_{3}^{1}\right) \\
& \vee\left(x_{1}^{1} \wedge x_{2}^{1} \wedge x_{3}^{1}\right), \\
= & \left(\neg x_{1} \wedge x_{2} \wedge x_{3}\right) \vee\left(x_{1} \wedge \neg x_{2} \wedge \neg x_{3}\right) \vee\left(x_{1} \wedge \neg x_{2} \wedge x_{3}\right) \vee \\
& \vee\left(x_{1} \wedge x_{2} \wedge x_{3}\right) .
\end{aligned}
$$

The last equation represents the canonical expression of ECA 184. 
It was pointed out in 3] that although ECA 184 belongs to (Wolfram's) Class I and so is considered to be one of the simplest, its "... evolution in fuzzy backgrounds indicates otherwise ... in particular the interaction between fuzziness and boolean cells creates complex structures. Even though it is difficult to theoretically explain this behavior, the experimental results quite clearly indicate that this is not a simple rule" [3], p. 112].

A CA on $[0,1]$ is said to be a continuous $C A$ if its domain is $[0,1] \times[0,1] \times$ $[0,1] \equiv[0,1]^{3}$ and its range is contained in the interval $[0,1]$. If $g$ is a general continuous CA on $[0,1]$ its fixed point set is by definition the collection of all points $x \in[0,1]$ such that $g(x, x, x)=x$. This set may consist of either a single point, a finite number of such points, or even the whole interval $[0,1]$. The latter occurs for the special CA dubbed FCA 184 that we study in this paper. We now turn to the concept of fuzzy CA's or, for brevity, FCA.

A Fuzzy CA is obtained by fuzzification of the local function of a Boolean CA: in the disjunctive normal form by redefining $(a \vee b)$ as $(a+b),(a \wedge b)$ as $(a b)$, and $(\neg a)$ as $(1-a)$. The usual fuzzification of the expression $a \vee b$ is $\max \{1, a+b\}$ so as to ensure that the result is not larger than 1 . Note, however, that taking $(a+b)$ for the CA fuzzification does not lead to values greater than 1 since the sum of all the expressions for rule 255 is 1 (i.e., $g_{255}(x, y, z)=1$ ), and so every (necessarily non-negative) partial sum must be bounded by 1 . Since every fuzzy rule is obtained by adding one or more of these partial sums it follows that every fuzzy rule is bounded below by 0 and above by 1 . We refer the reader to the many articles in the references provided below, e.g, [1] for a brief explanation of the basic principles behind the notion of fuzzy CA, or FCA as we call them here. Recall that in "fuzzifying" the DNF above (1), we replace $\neg x$ by $1-x, x \vee y$ by $x+y$, and $x \wedge y$ in (1) by their product, $x y$. By definition fuzzy CA are special cases of continuous CA.

Example 1. Referring to (1) and using the fuzzification procedure outlined above, we can write the form of FCA 184: Thus, starting from the disjunctive normal form of ECA 184,

$$
\begin{aligned}
g_{184}\left(x_{1}, x_{2}, x_{3}\right)= & \left(\neg x_{1} \wedge x_{2} \wedge x_{3}\right) \vee\left(x_{1} \wedge \neg x_{2} \wedge \neg x_{3}\right) \vee\left(x_{1} \wedge \neg x_{2} \wedge x_{3}\right) \vee \\
& \vee\left(x_{1} \wedge x_{2} \wedge x_{3}\right) \\
& \text { we fuzzify it to find } \\
= & \left(1-x_{1}\right) x_{2} x_{3}+x_{1}\left(1-x_{2}\right)\left(1-x_{3}\right)+x_{1}\left(1-x_{2}\right) x_{3}+x_{1} x_{2} x_{3}, \\
= & x_{1}-x_{1} x_{2}+x_{2} x_{3} .
\end{aligned}
$$

The transition rule derived from (2) for $\left(x_{1}, x_{2}, x_{3}\right) \in[0,1]^{3}$ in accordance with the fuzzification process defined here is denoted by FCA 184 (its two-state counterpart being given by (11))

We suggest that one of the reasons that this FCA deserves special attention is that its fixed-point set is a continuum, namely the whole closed interval $[0,1]$, (one of only 9 out of 255 such FCA, see [1] for the general theory). In addition, our purpose here is an attempt to explain previous insights in [3], at least theoretically. Generally speaking, although there are some exceptions, the evolution 
of a space-time diagram is undecidable, at least in the special case of boolean ECA. In this note we will actually determine the evolution of the space-time diagram of this FCA thereby answering an open question in [3], and thereby showing that its evolution is decidable in sharp contrast with the boolean case.

Table 1. Partial space-time diagram of FCA 184 for a two-cell initial string with $x_{0}^{0}=0.95, x_{1}^{0}=0.34$, in a homogeneous background of zeros. We are interested in what happens as we proceed down the diagram?

$\begin{array}{lccccccccc}0 & .95 & .34 & 0 & 0 & 0 & 0 & 0 & 0 & 0 \\ 0 & .3230 & .6270 & .34 & 0 & 0 & 0 & 0 & 0 & 0 \\ 0 & .2025 & .3337 & .4138 & .34 & 0 & 0 & 0 & 0 & 0 \\ 0 & .0676 & .2730 & .3363 & .2731 & .34 & 0 & 0 & 0 & 0 \\ 0 & .0184 & .1409 & .2731 & .3373 & .1803 & .34 & 0 & 0 & 0 \\ 0 & .0026 & .0543 & .1946 & .2418 & .3378 & .1190 & .34 & 0 & 0 \\ 0 & .0001 & .0130 & .0908 & .2292 & .2003 & .3380 & .0785 & .34 & 0 \\ 0 & .0000 & .0013 & .0327 & .1159 & .2510 & .1591 & .3382 & .0518 & .34\end{array}$

\section{The Space-Time Diagram of FCA 184 and its Evolution over Time}

Writing the canonical form of rule 184 as $g_{184}(x, y, z)=x-x y+y z$, its diagonal function $d(x) \equiv g_{184}(x, x, x)$ is given by solving the equation $d(x)=x$. Thus, every real number in $[0,1]$ is a fixed point (it is this fact that characterizes the other eight exceptional rules as well).

Next, for a single seed $a$ in a zero background observe that, by induction, $x_{n}^{n}=a$ for each $n \geq 1$, so that this rule is a right-shift. Clearly, for a single seed its dynamics are trivial. The difficulty occurs when we pass to the case of finite support/random initial strings of length greater than one.

Consider the case of two seeds, $a, b \in(0,1)$, in a zero background. We take it that $x_{0}^{0}=a, x_{1}^{0}=b$ (so that $x_{1}^{0}$ is to the right of $x_{0}^{0}$ ). Recall [1] that the symbol $x_{ \pm i}^{j}$ denotes the cell value $i$ cells to the right (or left) of $x_{0}^{0}$ at time $j$ (i.e, the $j^{t h}$-row). The convergence of the right-diagonals and the dynamics of our FCA along them is of special interest in this article. It is easy to see that $x_{n}^{n-1}=b$ for all $n \geq 1$, so that the limit of this sequence (or zeroth diagonal, $\mathcal{S}_{0}^{+}$), is $L_{0}^{+}=b$. Next, we note that the terms of the first right-diagonal, $\mathcal{S}_{1}^{+}$, are given by $x_{n}^{n}=a(1-b)^{n}$, a result that is easily verified by an induction argument. It follows that its limit, $L_{1}^{+}=0$, except in the special case where $b=0$, in which case this reduces to the single seed scenario already discussed above. Difficulties arise in the discussion bearing on the next diagonal, $\mathcal{S}_{2}^{+}$. Applying the technique presented in [1] to this situation, one that was successful in [10] and 11], we have that $L_{2}^{+}=g_{184}\left(L_{2}^{+}, L_{1}^{+}, L_{0}^{+}\right)=g_{184}\left(L_{2}^{+}, 0, b\right)=L_{2}^{+}$. Thus, no a priori information regarding $L_{2}^{+}$is obtainable using the methods in [1], as they stand (and this is characteristic of these few exceptional FCA). Thus, a new technique 
is needed and the following method serves as a blueprint that also applies to the remaining exceptional FCA. Observe that by definition,

$$
x_{n}^{n+1}=x_{n-1}^{n}\left(1-a(1-b)^{n}\right)+a b(1-b)^{n},
$$

for $n \geq 1$. Defining $a b y_{n} \equiv x_{n}^{n+1}$ we get a simple recurrence relation for $y_{n}$, i.e.,

$$
y_{n}=y_{n-1}\left(1-a(1-b)^{n}\right)+(1-b)^{n}, \quad y_{0}=1,
$$

where the solution, in this two-seed case, is given by

$$
y_{k}=\prod_{j=0}^{k-1}\left(1-a(1-b)^{j+1}\right)\left\{1+\sum_{i=0}^{k-1} \frac{(1-b)^{i+1}}{\prod_{j=0}^{i}\left(1-a(1-b)^{j+1}\right)}\right\}
$$

for $k \geq 1$. Furthermore, since $\left|y_{n+1}-y_{n}\right| \leq(1-b)^{n+1}$ it follows that the sequence $\left\{y_{n}\right\}$ is a Cauchy sequence. Its limit $L_{2}^{+}$, found by passing to the limit as $k \rightarrow \infty$ in (3) and rearranging terms, is given by

$$
L_{2}^{+}=\prod_{j=0}^{\infty}\left(1-a(1-b)^{j+1}\right)+\sum_{i=0}^{\infty}(1-b)^{i+1} \prod_{j=i+1}^{\infty}\left(1-a(1-b)^{j+1}\right),
$$

where the infinite products are convergent by virtue of their form. Note that $L_{2}^{+} \neq 0$ for $a, b \in(0,1)$. The continuity of the transition rule for FCA 184 now implies that

$$
L_{3}^{+}=g_{184}\left(L_{3}^{+}, L_{2}^{+}, L_{1}^{+}\right)
$$

a relation that forces $L_{3}^{+}=0$. The existence of $L_{4}^{+}$is found by appealing to a recurrence relation once again but this time of the form $z_{n} \equiv x_{n}^{n+2}$,

$$
z_{n}=z_{n-1}\left(1-a b y_{n}\right)+a^{2} y_{n} b(1-b)^{n},
$$

whose solution can be written once again but is omitted here for brevity. Continuing in this way one can show by induction that $L_{2 k-1}^{+}=0, L_{2 k-2}^{+} \neq 0$, for $k \geq 1$, found by solving first order recurrence relations leading to solutions involving complicated infinite products in the variables $a, b$. Clearly, this method leads to an algorithm for producing the limits $L_{k}^{+}$of the right-diagonals of the space-time diagram of FCA 184 . Note that the left diagonals produce zero limits at infinity.

The case of three or more seeds is similar to the two-seed case and is sketched for completeness. We assume a homogeneous background of zeros in which there is embedded the initial string $\ldots 0,0,0, x_{-m}^{0}, \ldots, x_{0}^{0}, x_{1}^{0}, 0,0,0, \ldots$ of non-zero cell values. Then, as before, $L_{0}^{+}=x_{1}^{0}$ and $x_{n}^{n}=x_{0}^{0}\left(1-x_{1}^{0}\right)^{n}$ so that, once again, $L_{1}^{+}=0$. Taking $x_{0}^{0} x_{1}^{0} y_{n} \equiv x_{n}^{n+1}$ we get a corresponding recurrence relation for $y_{n}$,

$$
y_{n}=y_{n-1}\left(1-x_{0}^{0}\left(1-x_{1}^{0}\right)^{n}\right)+\left(1-x_{1}^{0}\right)^{n}, \quad y_{0}=1 \text {. }
$$

The solution of the preceding equation is representable as a quantity analogous to (3) above so that the existence of $L_{2}^{+}$is guaranteed and is an expression 
similar to (4) involving infinite products. As in the two-seed case, the quantities $L_{2 k-1}^{+}=0, L_{2 k-2}^{+} \neq 0$, for $k \geq 1$. This completes the analysis of the finite initial configuration case.

As for vertical columns/sequences of the form $x_{0}^{j}, x_{1}^{j}$, etc. for $j \geq 1$, it is unlikely that these vertical sequences will converge at all due to the alternating nature of zero limits (i.e., $L_{2 k-1}^{+}=0$ ) along right-diagonals. Specifically, if one can show that the limit of the limits, $L_{2 k-2}^{+} \rightarrow 0$, as $k \rightarrow \infty$ (which we cannot prove) then one can infer that the vertical sequences will all converge to zero. Numerical evidence though seems to support this claim (cf., Table 1). On the other hand, if

$$
\liminf _{k \rightarrow \infty} L_{2 k-2}^{+}>0,
$$

then for each $i \geq 0, \lim _{k \rightarrow \infty} x_{i}^{k}$ does not exist.

\section{Conclusion}

We have shown that FCA 184, one of six exceptional fuzzy cellular automata [1] whose set of fixed points is a continuum, has complicated behavior at infinity but does not lend itself to complexity, thus answering a question raised in [3]. Indeed, the asymptotic nature of its space-time diagram is deterministic and apparently non-chaotic, the evolution from any finite random initial string of fuzzy values in the open unit interval being decidable, as opposed to its boolean counterpart.

\section{Acknowledgments}

This research is partially supported by an NSERC Canada Research Grant to the first author.

\section{References}

1. Angelo B. Mingarelli, The global evolution of general fuzzy cellular automata, Int. J. Unconventional Comp., Vol. X, (2005), 1-24.

2. S. Wolfram, A New Kind of Science, Wolfram Media, Champaign, Il., 2002.

3. G. Cattaneo, P. Flocchini, G. Mauri, C. Quaranta Vogliotti, N. Santoro, Cellular automata in fuzzy backgrounds, Physica D 105 (1997), 105-120

4. C. A. Reiter, Fuzzy automata and life, Complexity, 7 (3) (2002), 19-29.

5. P. Maji, R. Nandi, P. Chaudhuri, Design of fuzzy cellular automata (FCA) based pattern classifier, in Fifth International Conference on Advances in Pattern Recognition, ICAPR-2003, December 10-13, Calcutta, India. To appear.

6. G. Cattaneo, P. Flocchini, G. Mauri, and N. Santoro, Fuzzy cellular automata and their chaotic behavior, in Proc. International Symposium on Nonlinear Theory and its Applications, Hawaii, IEICE Volume 4, (1993) 1285-1289

7. P. Flocchini, F. Geurts, A. Mingarelli, N. Santoro, Convergence and aperiodicity in fuzzy cellular automata: revisiting rule 90, Physica D, 42 (2000), 20-28. 
8. T. Gramss, S. Bornholdt, M. Gross, M. Mitchell, T. Pellizzari, Computation in cellular automata: A selected review in Nonstandard Computation: Weinheim: VCH Verlagsgesellschaft (1998), 95-140.

9. S. Wolfram, Theory and Applications of Cellular Automata, World Scientific, Singapore, 1986.

10. Angelo B. Mingarelli, Fuzzy rule 110 dynamics and the golden number, WSEAS Trans. Computers, 2 (4) (2003), 1102-1107.

11. Angelo B. Mingarelli and Elzbieta Beres, The dynamics of fuzzy cellular automata: Rule 30, WSEAS Trans. Circuits and Systems, (10) 3, 2211-2216. 